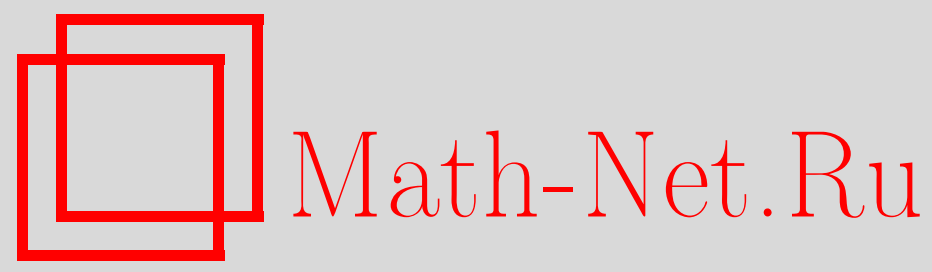

А. А. Летуновский, Цикловые индексы автомата, Дискрет. матем., 2013, том 25, выпуск 4, 24-29

DOI: https://doi.org/10.4213/dm1254

Использование Общероссийского математического портала Math-Net.Ru подразумевает, что вы прочитали и согласны с пользовательским соглашением http://www . mathnet.ru/rus/agreement

Параметры загрузки:

IP: 18.207.199.55

26 апреля 2023 г., 17:01:23 


\title{
Цикловые индексы автомата
}

\author{
() 2013 г. А. А. Летуновский
}

Вводится понятие цикловых индексов автомата как пары положительных чисел $(b, q)$, эффективно вычисляемых по автомату. Показано, что множество периодов константных автоматов, выразимых суперпозициями данного автомата, булевых функций и задержки - суть делители членов геометрической прогрессии $b, b q, b q^{2}, \ldots$ Отсюда следуют алгоритм проверки выразимости константных автоматов, а также теорема о проверке выразимости множества всех автоматов с ограниченным числом состояний.

Пусть $E_{2}=\{0,1\}$, функции вида $g: E_{2}^{n} \rightarrow E_{2}$ называются булевыми функциями, их множество обозначается через $\mathbf{P}_{2}$. Пусть $E_{2}^{\infty}-$ множество всех сверхслов вида $a(1) a(2) \ldots$, где $a(j) \in E_{2}, j=1,2, \ldots$ Через $\mathbf{N}$ обозначим множество натуральных чисел. Для $k, l \in \mathbf{N}$ будем обозначать через $k \mid l$ то, что $k$ делит $l$. Пусть

$$
f:\left(E_{2}^{\infty}\right)^{n} \rightarrow\left(E_{2}^{\infty}\right)^{m}
$$

- автоматная функиия (а-функиия), т.е. она задается рекуррентно соотношениями (1)

$$
\left\{\begin{array}{l}
q_{1}(1)=q 0_{1}, \\
\ldots \\
q_{s}(1)=q 0_{s}, \\
q_{1}(t+1)=\phi_{1}\left(q_{1}(t), \ldots, q_{s}(t), a_{1}, \ldots, a_{n}\right), \\
\ldots \\
q_{s}(t+1)=\phi_{s}\left(q_{1}(t), \ldots, q_{s}(t), a_{1}, \ldots, a_{n}\right), \\
b_{1}(t)=\psi_{1}\left(q_{1}(t), \ldots, q_{s}(t), a_{1}, \ldots, a_{n}\right), \\
\ldots \\
b_{m}(t)=\psi_{m}\left(q_{1}(t), \ldots, q_{s}(t), a_{1}, \ldots, a_{n}\right) .
\end{array}\right.
$$

Вектор $q=\left(q_{1}, \ldots, q_{s}\right)$ задает состояние $a$-функции $f, q_{0}$ - её начальное состояние, буквы $a=\left(a_{1}, a_{2}, \ldots, a_{n}\right)$ и $b=\left(b_{1}, \ldots, b_{m}\right)$ называются входной и выходной буквами, а сверхслова $a(1) a(2) \ldots$ и $b(1) b(2) \ldots$ - входными и выходными сверхсловами, соответственно. Вектор-функции $\phi$ и $\psi$ называются функциями переходов и выходной функцией, соответственно, а шестерка

$$
\left(E_{2}^{n}, E_{2}^{s}, E_{2}^{m}, \phi, \psi, q 0\right)
$$

- автоматом, порождающим функцию $f$. Далее в тексте мы иногда будем использовать для автомата обозначение $(A, Q, B, \phi, \psi, q 0)$, при этом предполагая что $A \subseteq E_{2}^{n}, Q \subseteq E_{2}^{s}, B \subseteq E_{2}^{m}$. Автомат называется автоматом Медведева, если $B=Q, \psi(a, q) \equiv q$. 
Обычным образом доопределим функции $\phi$ и $\psi$ на слова [1]:

$$
\begin{gathered}
\phi(q, a(1) \ldots a(t))=\phi(\phi \ldots \phi(q, a(1)), \ldots, a(t-1)), a(t)), \\
\psi(q, a(1), \ldots, a(t))=\psi(\phi(q, a(1)), \ldots, a(t-1)), a(t))
\end{gathered}
$$

и определим рекурсивно функцию

$\bar{\psi}(q, a(1), \ldots, a(t))=\bar{\psi}(q, a(1), \ldots, a(t-1)) \psi(\phi(q, a(1) \ldots a(t-1)), a(t))$.

Класс всех $a$-функций обозначим через $\mathbf{P}$. Класс всех $a$-функций с не более чем $d$ состояниями обозначим $\mathbf{P}^{d}$.

В этом классе обычным образом введем операции суперпозиции. Для суперпозиции будем использовать модификации операций из [2]:

$$
\begin{aligned}
& (\eta f)\left(x_{1}, x_{2}, . ., x_{n}\right)=f\left(x_{2}, x_{3}, \ldots, x_{n}, x_{1}\right), \\
& (\varepsilon f)\left(x_{1}, x_{2}, . ., x_{n}\right)=f\left(x_{2}, x_{1}, x_{3}, \ldots, x_{n}\right), \\
& (\varpi f)\left(x_{1}, x_{2}, . ., x_{n}\right)=f\left(x_{1}, x_{3}, . ., x_{n}\right), \\
& (\delta f)\left(x_{1}, x_{2}, . ., x_{n}\right)=f\left(x_{1}, x_{2}, . ., x_{n+1}\right), \\
& (f * g)\left(x_{1}, x_{2}, . ., x_{m+n-1}\right)=f\left(g\left(x_{1}, \ldots, x_{m}\right), x_{m+1}, \ldots, x_{m+n-1}\right) .
\end{aligned}
$$

Пусть $R \subseteq \mathbf{P}$; обозначим через $[R]$ множество $a$-функций, получающихся из $R$ с помощью операций суперпозиции. Рассматривая системы автоматов, будем считать без ограничения общности, что $R$ состоит из одного автомата, так как задачу выразимости для нескольких автоматов можно свести к задаче для одного автомата, являющегося их параллельным соединением.

Автоматную функцию $G_{0}$, задаваемую уравнениями

$$
\left\{\begin{array}{l}
q(1)=0, \\
q(t+1)=a(t), \\
b(t)=q(t),
\end{array}\right.
$$

назовём автоматной функиией "задержки".

Пусть $<R>=\left[R \cup\left\{\mathbf{P}_{2}, G_{0}\right\}\right]$.

Назовем автомат групповым, если все $\phi_{a}(q)=\phi(a, q), a \in A$, являются биекциями на $Q$.

Назовем автоматную функцию, не зависящую от входа, константной автоматной функцией. Множество всех константных автоматных функций обозначим К. Без ограничения общности константную автоматную функцию можно отождествить со сверхсловом, которое является его выходом.

Пусть сверхслово $\beta$ можно представить в виде $\beta=\gamma \alpha^{\infty}$. Выберем из всех таких представлений то, в котором $\gamma$ и $\alpha$ имеют наименьшую длину. Для выбранного представления назовем $\gamma$ наименьшим предпериодом сверхслова $\beta$, а $\alpha$ - наименьшим периодом сверхслова $\beta$, а слова вида $\underbrace{\alpha \alpha \ldots \alpha}_{n}$ будем также называть периодами сверхслова $\beta$. Обозначим $|\alpha|$ длину слова $\alpha$.

Для множества константных автоматных функций $K^{\prime} \subseteq K$ обозначим через $\Theta\left(K^{\prime}\right)$ множество длин минимальных периодов сверхслов $\left\{\beta_{K_{i}}: K_{i} \in K^{\prime}\right\}$. Для случая одного слова $\beta=\gamma \alpha^{\infty}$ будем считать, что $\Theta(\beta)=|\alpha|$.

Нашей задачей будет описание множества $\Theta(<R>\cap K)$ для произвольного множества $R$.

Лемма 1. Пусть $K_{1}, K_{2} \in K$, причем $\Theta\left(K_{2}\right) \mid \Theta\left(K_{1}\right)$. Тогда $K_{2} \in<K_{1}>$. 
Лемма 1 показывает, что, имея в замыкании хотя бы одну константу периода $l$, мы можем выразить любую константу периода, делящего $l$.

Замечание 1. Если $|\Theta(<R>\cap K)|<\infty$, то

$$
\Theta(<R>\cap K)=\{l \in N: l \mid \max (\Theta(<R>\cap K))\} .
$$

Замечание 2. Если $K_{1}, K_{2} \in K$, mo

$$
\max \left(\Theta\left(<\left\{K_{1}\right\} \cup\left\{K_{2}\right\}>\right)\right)=\operatorname{HOK}\left(\max \left(\Theta\left(K_{1}\right)\right), \max \left(\Theta\left(K_{2}\right)\right)\right) .
$$

Фактически конечное $K^{\prime} \subset K$ имеет множеством периодов делители одного числа $l=\max \left(\Theta\left(K^{\prime}\right)\right)$. Тем самым всякое конечное множество константных функций таково, что $<K^{\prime}>=\left\{\beta_{l}\right\}$, где $\beta_{l}-$ константа периода $l$.

Из известной леммы об удлинении периода констант автоматом [3] следует, что множество длин периодов констант, выразимых автоматом, имеет вид $2^{l_{1}} 3^{l_{2}} \ldots p_{i}^{l_{i-1}}$, где $p_{i}$ - простые числа, $p_{i} \leqslant|Q|$, а $l_{i}$ - натуральные числа.

Для некоторого автомата $M$ и произвольного слова $\alpha \in A^{*}$ обозначим через $s_{\alpha}=$ $\phi(q, \alpha)$ - подстановку на множестве состояний, задаваемую этим словом, через $\pi_{\alpha}$ - разбиение множества состояний $Q$ на классы отличимости $Q_{1}, \ldots, Q_{s}$ этим словом. Состояния $q_{i}$ и $q_{j}$ принадлежат одному классу отличимости, если $\bar{\psi}\left(q_{i}, \alpha\right)=\bar{\psi}\left(q_{j}, \alpha\right)$.

Обозначим $d_{\alpha}=\left(s_{\alpha}, \pi_{\alpha}\right)$. Пусть $D_{l}=\left\{d_{\alpha},|\alpha|=l\right\}$.

Рассмотрим последовательность $n_{1}, n_{2}, \ldots$ натуральных чисел, связанную с автоматом $M$, где $n_{i+1}$ получается из $n_{i}$ следующим рекурсивным способом.

Пусть $c_{i}=\left\{\alpha_{i}\right\}$ - множество сверхслов с длиной периода $l \mid n_{i}$. Рассмотрим множество $M\left(c_{i}\right)$ выходных сверхслов автомата $A$ после подачи на него слов из $c_{i}$. Очевидно, что $M\left(c_{i}\right)$ конечно. Тогда положим $n_{i+1}=H O K\left(\Theta\left(M\left(c_{i}\right)\right)\right)$. Из замечаний 1,2 следует, что $n_{i}$ - максимальная длина периода констант, выразимых схемой глубины $i$.

По построению $n_{i} \mid n_{i+1}$. Далее мы докажем, что $m_{i}=\frac{n_{i+1}}{n_{i}}-$ периодическая последовательность.

Лемма 2. Если $D_{l}=D_{m}$ для некоторьх $l, m$, mо $D_{l k}=D_{m k}$ для любого $k \in N$.

Доказательство. Для доказательства включения $D_{l k} \subseteq D_{m k}$ покажем, что для каждого $\alpha,|\alpha|=l k$ существует такое $\alpha_{1},\left|\alpha_{1}\right|=m k$, что $d_{\alpha}=p_{\alpha_{1}}$. Пусть $\alpha=\beta_{1} \ldots \beta_{k}$, где $\left|\beta_{i}\right|=l$. Тогда $s_{\alpha}=s_{\beta_{1}} * \ldots * s_{\beta_{k}}$, а $\pi_{\alpha}$ будет получаться сначала как разбиение, задаваемое $\beta_{1}$, затем как измельчение этого разбиения разбиением, задаваемым словом $\beta_{1} \beta_{2}$, и т.д. Для каждого $p_{\beta_{i}} \in D_{l}$ существует такое $p_{\gamma_{i}} \in D_{m}$, что $p_{\beta_{i}}=p_{\gamma_{i}}$. Рассмотрим слово $\alpha_{1}=\gamma_{1} \ldots \gamma_{k}$. Для него выполнено $p_{\alpha}=p_{\alpha_{1}}$. Действительно $s_{\alpha 1}=s_{\gamma 1} * \ldots * s_{\gamma k}$, а $\pi_{\alpha_{1}}$ будет получаться сначала как разбиение, задаваемое $\gamma_{1}$, затем как измельчение этого разбиения разбиением $\gamma_{1} \gamma_{2}$ и т.д.

Таким образом, $D_{l k} \subseteq D_{m k}$; обратное включение доказывается аналогично. Лемма доказана.

Лемма 3. Последовательность $m_{i}$ периодична.

Доказательство. Пусть нашлись такие $n_{i}, n_{j}$, что $D_{n_{i}}=D_{n_{j}}$. Из построения $m_{i}$ следует, что $m_{i}$ - это фактически длина максимального удлинения, получаемого при подаче слов длины $n_{i}$ на автомат $A$ и затем взятия HOK этих удлинений. Очевидно, что множество удлинений однозначно задается множеством $D_{n_{i}}$. Таким образом, $m_{i}=m_{j}$, и из леммы 2 следует, что $D_{n_{i+1}}=D_{n_{j+1}}$, а тогда $m_{i+1}=m_{j+1}$, и лемма доказана. 
Теперь определим иикловые индексы автомата через алгоритм их вычисления.

1. Вычисляем последовательность $\left(n_{i}, D_{i}\right)$ до тех пор, пока не найдутся такие $j<i$, что $D_{n_{i}}=D_{n_{j}}$.

2. Назовем $b=n_{j}$ безусловным цикловым индексом автомата, $q=\frac{n_{i}}{n_{j}}-$ главным цикловым индексом автомата.

Теорема 1. Если $R$ - конечное множество автоматных функиий, то

$$
\Theta(<R>\cap K)=\bigcup_{i=1}^{\infty}\left\{t \mid b q^{i}\right\}
$$

где $b, q-$ иикловые индексы системы автоматов $R$.

Доказательство. Пусть константа $\beta$ содержится в замыкании нашего множества; рассмотрим схему, выражающую данную константу. Пусть глубина этой схемы равна $L$. Тогда по построению последовательности $n_{i}$ и из леммы об удлинении периодов констант следует что $\Theta(\beta) \mid n_{L}$.

Рассмотрим произвольную константу периода $b q^{i}$. По лемме 2 , а также по построению чисел $b$ и $q$, данная константа выразима схемой подходящей глубины. Теорема доказана.

Приведем несколько утверждений, непосредственно следующих из теоремы 1.

Теорема 2. Если $R$ - конечное множество автоматных функиий и $\beta$ - константная автоматная функиия, то существует алгоритм, позволяющий проверить свойство $\beta \in<R>$.

Следствие 1. Если $R$ - конечное множество автоматных функиий, то существует алгоритм, позволяющий проверить свойство $|\Theta(<R>\cap K)|<\infty$.

Теорема 3 (Необходимое условие выразимости). Пусть $R_{1}, R_{2}$ - конечные множества автоматов и $R_{2} \in\left[R_{1}\right]$; пусть $b_{1}, q_{1}, b_{2}, q_{2}$ - иикловые индексы $R_{1} u R_{2}$ соответственно. Тогда

$$
\bigcup_{i=1}^{\infty}\left\{t \mid b_{2} q_{2}^{i}\right\} \subseteq \bigcup_{i=1}^{\infty}\left\{t \mid b_{2} q_{2}^{i}\right\}
$$

$u$

$$
\Theta\left(<R_{2}>\cap K\right) \subseteq \Theta\left(<R_{1}>\cap K\right) .
$$

В работе [4] Д.Н. Бабин ввел определение "копирования"одного автомата другим и доказал "Лемму о копировании".

Определение 1 ([4]). Пусть $f$ и $g$ - автоматы с одинаковым числом входов и одинаковым числом выходов. Скажем, что автоматная функция $g$ копирует автоматную функцию $f$, если найдутся такие натуральные $n, j, s(n \leqslant j)$, что для любого $l=0,1,2, \ldots$ и любой входной последовательности достаточной длины значение автоматной функции $g$ в момент $j+s l$ совпадает со значением автоматной функции $f$ в момент $n+s l$, т.е. $f(n+s l)=g(j+s l)$. 
Пусть $K_{s}$ - константный автомат $(\underbrace{1 \ldots 0}_{s})^{\infty}$.

Заметим что свойство копирования автоматной функции $f$ автоматной функцией $g$ алгоритмически проверяемо.

Лемма о копировании [4]. Если $f$ - автоматная функиия Медведева и $g$ копирует $f$ с параметрами $n, j, s$, то $f \in<\in K_{s}>$.

Техника цикловых индексов позволяет усилить данную лемму.

Лемма 4. Если $f$ - автоматная функиия Медведева, $g$ копирует $f$ и выполнено условие $|\Theta(<g>\cap K)|=\infty$, mо $f \in<g\rangle$.

Автоматную функцию Медведева $F_{2}$, с функциями переходов и выходов

$$
\begin{array}{ll}
\phi\left(q_{1}, 00\right)=q_{1}, & \phi\left(q_{2}, 00\right)=q_{2}, \\
\phi\left(q_{1}, 01\right)=q_{1}, & \phi\left(q_{2}, 01\right)=q_{1}, \\
\phi\left(q_{1}, 10\right)=q_{2}, & \phi\left(q_{2}, 10\right)=q_{2}, \\
\phi\left(q_{1}, 11\right)=q_{2}, & \phi\left(q_{2}, 11\right)=q_{1}
\end{array}
$$

назовём универсалъным автоматом с 2 состояниями.

Теорема 4. Если $R$ - конечное множество автоматов, то свойство $<R \cup F_{2}>\supseteq$ $\mathbf{P}^{d}$ алгоритмически проверяемо при всех натуралъных $d$.

Доказательство теоремы 4 приведено в работе [9] и основано на утверждениях, полученных в данной работе, а также в работах $[6,7,8]$.

Работа выполнена на кафедре МаТИС механико-математического факультета МГУ им. Ломоносова под руководством профессора Д.Н. Бабина.

\section{Список литературы}

1. Кудрявцев В. Б., Алешин С. В., Подколзин А. С., Введение в теорию автоматов. Наука, Москва, 1985.

2. Мальцев А. И., Итеративные алгебры Поста. Изд-во ИМ СО АН СССР, Новосибирск, 1976.

3. Loomis Jr H. H., Completeness of sets of delayed logic devices. IEEE Trans. Electronic Computers (1965) EC-14, 157-172.

4. Бабин Д. Н., О полноте двухместных автоматных функций относительно суперпозиции. Дискрет. матем. (1989) 1, №4, 423-431.

5. Летуновский А. А., О выразимости константных автоматов суперпозициями. Интеллектуальные системы (2009) 13, №1-4, 397-406.

6. Алешин С. В., Об одном следствии теоремы Крона-Роудза. Дискрет. матем. (1999) 11, №4, 101-109.

7. Бабин Д. Н., О суперпозициях ограниченно детерминированных функций ограниченного веса. Логико-алгебраические конструкиии (1992) 21-27. 
8. Летуновский А. А., О выразимости суперпозициями автоматов с разрешимыми группами. Интеллектуальные системы (2010) 14, №1-4, 379-392.

9. Летуновский А. А., О задаче выразимости автоматов относительно суперпозиции для систем с фиксированной добавкой. Интеллектуальные системы (в печати).

Статья поступила 09.11.2012. 